\title{
A Third Ascending Hypothalamopetal Pathway
}

\author{
George WolF
}

Division of Natural Sciences, State University of New York

Purchase, Newe York 10577

AND

LeO V. DiCARA

Department of Psychiatry, Unizersity of Michigan, Ann Arbor, Michigan 48103

Reccived May 26, 1971

\begin{abstract}
Electrolytic lesions centered in the reticular formation or partial hemisections of the brain stem were induced stereotaxically at various levels from the medulla to the diencephalon. Nauta and Fink-Heimer methods were used to reveal degenerated fibers ascending through the upper brain stem. Degenerated fibers coursing rostrally through the subthalamus and ventrally to enter the lateral hypothalamus at tuberal levels were observed after lesions at all levels rostral to the medulla. The trajectory of this ascending pathway through the midbrain and diencephalon was distinct from that of the dorsal longitudinal fasciculus which is medial and that of the medial forebrain bundle which is ventral. Several lines of evidence suggest the hypothesis that this pathway is a component of the ascending reticular system which carries sensory information into the hypothalamus for correlation with hypothalamic visceral and behavioral functions.
\end{abstract}

\section{Introduction}

Fibers originating in midline and paramedian tegmental nuclei and ascending to the hypothalamus through the dorsal longitudinal fasciculus and the medial forebrain bundle have been the subject of several studies and are well documented and widely known (10). A third topographically distinct group of fibers ascending to the hypothalamus has been observed by several workers using Golgi $(16,17)$, Nauta $(13,20,21)$, or fluorescence (7) methods. ${ }^{2}$ This third pathway has not yet been the subject of intensive

1 Supported by Grants 70684 and 69-797 from the American Heart Association and Grant MH 19172 from USPHS, and done during first author's tenure of an Established Investigatorship from the American Heart Association. Portions of this work have been presented in preliminary form $(20,21)$ and read at the 1971 convention of the American Association of Anatomists.

2 Dr. W. J. Nauta has informed us that the subthalamic-hypothalamic fibers which are described briefly in his review paper on hypothalamic connections (10) degenerate. after small lesions of Forel's fields. Thus, this may be considered as additional independent evidence for the existence of this pathway in another species (cat). 
anatomical study and the establishment of its existence as a separate ascending hypothalamopetal system requires further anatomical analysis and documentation. However, the data thus far concur rather well to indicate that the fibers course rostrally with the central tegmental tract to pass through the subthalamus and finally enter the lateral hypothalamus from above. The data from Golgi preparations provide strong evidence that the cells of origin are in the reticular formation. The degeneration data, although less convincing since reticular lesions inevitably destroy fibers of passage as well as local cell bodies, are entirely congruent with the Golgi data in this regard.

To facilitate discussion we shall refer to the above group of fibers as the reticulohypothalamic pathway. Also, we are using the term "central tegmental tract" to include fibers of diverse origin passing diffusely through the reticular formation. As depicted in the cat by Nauta and Kuypers (11), the central tegmental tract occupies most of the cross-sectional area of the reticular formation in the upper brain stem. The purpose of the present paper is to demonstrate the distinct diencephalic trajectory of the reticulohypothalamic pathway, to provide further information on the brain stem levels at which it takes origin, and to review evidence suggesting the hypothesis that it has the function of conveying sensory information to the hypothalamus.

\section{Methods}

Twenty-two adult male Sprague-Dawley rats were used. They were placed in a stereotaxic instrument under Nembutal anesthesia. Lesions 1-2 $\mathrm{mm}$ in diameter were made by passing anodal direct current through stainless-steel electrodes insulated except for the tip. Such lesions were placed unilaterally in the brain stem at the level of the caudal diencephalon of twod, midbrain of eight, caudal pons of two, and upper medulla of two rats. In order to maximize the chances of observing any degenerating hypothalamic fibers which might originate diffusely in the lower brain stem, broad unilateral transections were produced by lowering a $2-\mathrm{mm}$ wide spatula into the caudal pons of five, or upper medulla of three rats.

The rats were killed 3-6 days after the lesions had been made, and the brains were removed after perfusion with phosphate-buffered formalin solution. Frozen sections of 20 brains were cut in frontal planes, and saggital sections were prepared from brains of two rats with midbrain lesions. The sections were cut at $25 \mu$ thickness. In some cases alternate sections through the larger lesions were cut at $100 \mu$ to minimize distortion and fragmentation. The $100-\mu$ sections and selected $25-\mu$ sections were stained by either Kluver-Barrera or cresyl violet methods. Degenerating fibers were stained by the Nauta method (with uranyl nitrate pretreatment) and by 
the Fink-Heimer method to help identify terminal fields. Some of these sections were bleached with potassium ferricyanide and counterstained with cresyl violet to facilitate identification of nuclei. Degeneration observed microscopically was plotted on projection drawings at $10 \times$ magnification.

\section{Results}

Figure 1 shows a representative mesencephalic lesion with the resultant degeneration in a saggital plane through the far lateral hypothalamus. Consider first the pattern of degeneration in the diencephalon. A clear clorsoventral bifurcation of the central tegmental tract occurs at the mesodiencephalic junction as described by other workers $(11,16)$. The ventral branch passes rostrally through the sulthalamus with terminations throughout its course. $\Lambda$ number of the more ventral subthalamic fibers

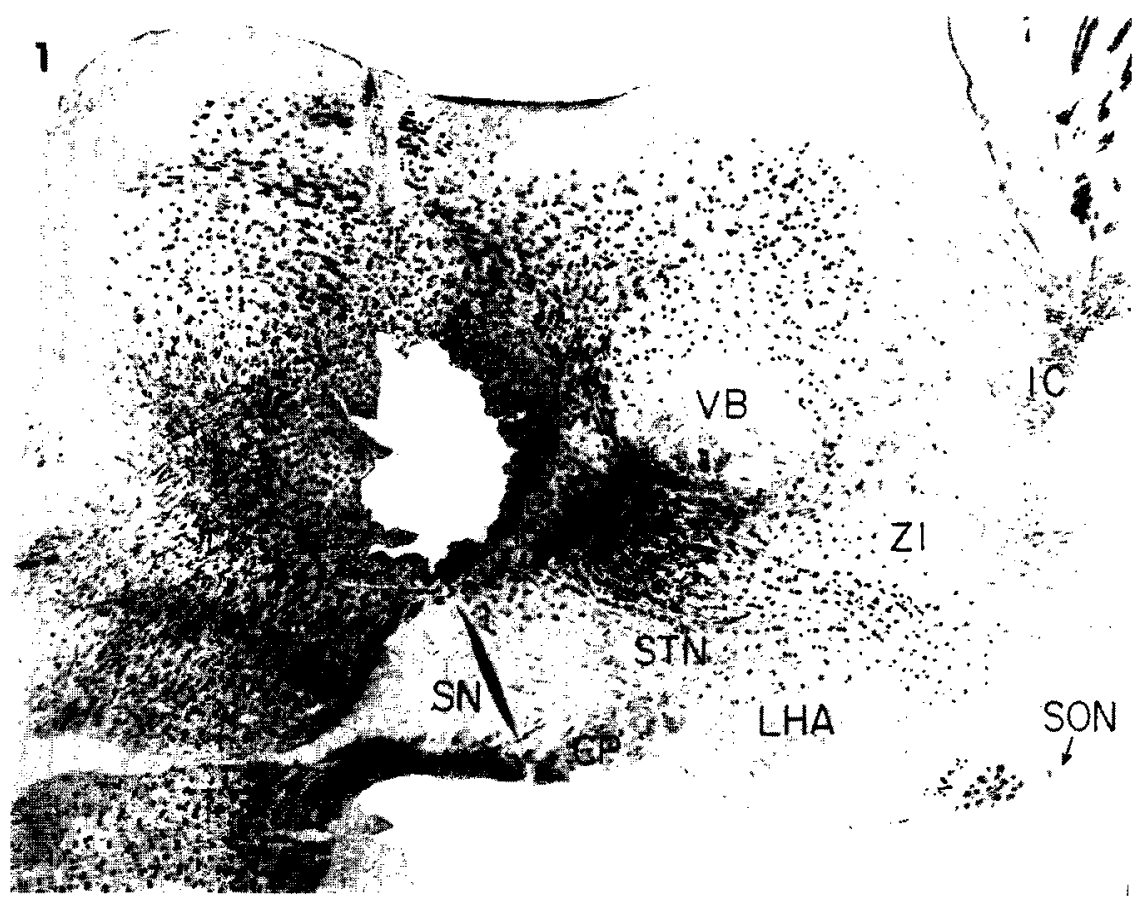

FIG. 1. Degeneration after a large mesencephalic lesion plotted schematically as dashes (degenerated fiber bundles) and dots (degenerated single fibers and terminals) on a camera lucida drawing of a saggital section through the far lateral hypothalamus. The section was stained by the Nauta method and counterstained with cresyl violet. Abbreviations: CP, cerebral peduncle; IC, internal capsule; I.HA, lateral hypothala. mic area; SN, substantia nigra; SON, supraoptic nucleus; STN, subthalamic nucleus; $\mathrm{VB}$, ventrobasal complex; ZI, zona incerta. Drawings showing the pathway in frontal planes are given in Ref. 20. 
reach the lateral hypothalamus at tuberal levels and appear to terminate there. More lateral and dorsal subthalamic fibers enter the entopeduncular nucleus and globus pallidus (lateral to the plane of Figure 1). The distribution of the dorsal branch of the central tegmental tract in the thalamus corresponds to previous descriptions $(11,16)$. Except for some degenerated fibers in the ventral supraoptic commissures all hypothalamic degeneration was ipsilateral regardless of the level or size of the lesion.

The left side of Fig. 2 shows a representative lesion at each of the four brain-stem levels and the right side shows the corresponding lateral hypothalamic degeneration. The larger lesions generally destroyed the major cross-sectional area of the reticular formation. The two caudal diencephalic lesions were similar in size and located at the level of bifurcation of the central tegmental tract (Fig. 2A). Both lesions disjoined the central tegmental tract almost totally while sparing periventricular and caudal hypothalamic structures.

The mesencephalic lesions varied more in size but the locus was consistantly within the tegmentum at the level of the superior colliculus as shown in Figs. 1 and 2B. Although all the mesencephalic lesions encroached on one or more peripheral structures (such as tectum, geniculate body, central gray matter, or red nucleus), each known peripheral structure which might give rise to the hypothalamopetal fibers was spared by at least one of the mesencephalic lesions as well as by the pontine lesions (Fig. 2C) which also induced hypothalamic degeneration.

The pontine and bulbar lesions (both electrolytic and mechanical) were made large in order to maximize the chances of inducing hypothalamic degeneration which was quite sparse when the lesions were at lower brain stem levels. While the lesions destroyed most of the cross-sectional area of the reticular formation, variations in mediolateral placement (Fig. 2C, D) resulted in differences in the encroachment upon peripherally adjacent nuclei.

The right side of Fig. 2 is intended to convey our impression of the relative density of hypothalamic degeneration gained from intensive study of the 22 specimens each stained with several degrees of suppression. In spite of the substantial variability of the data, there was a gross diminution in the apparent density of hypothalamic degeneration with more caudal lesion placement. The diminution was specially notable from the mesencephalic (Fig. 2B) to the caudal pontine (Fig. 2C) lesions which were spaced furthest apart. When the lesions were below the pons there was essentially no significant degeneration to be found in the hypothalamus although the subthalamic and thalamic degeneration which was always more dense was still clearly apparent.

The variations in lesion placement provide data relevant to the proposed 

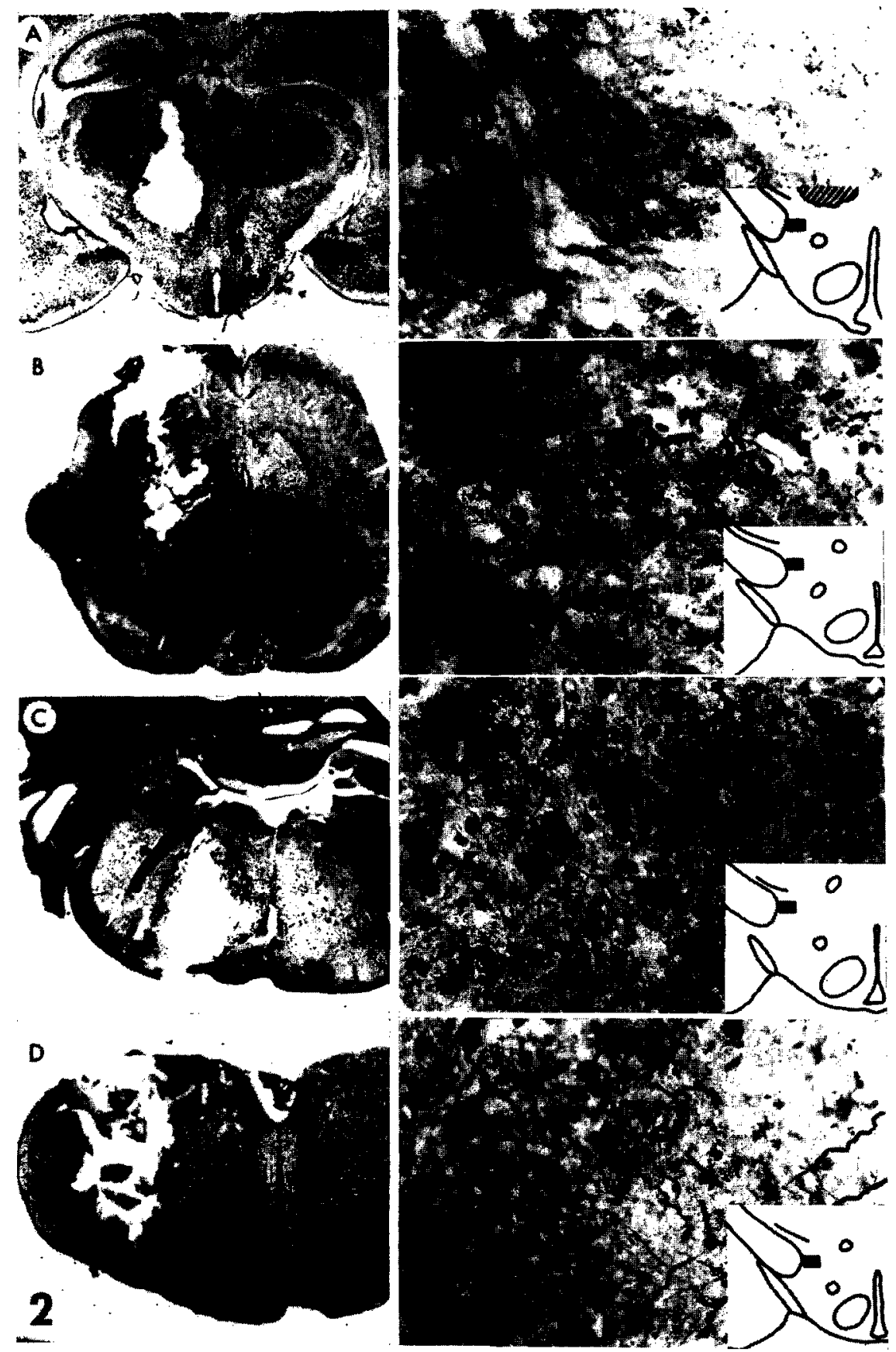

FII. 2. Photomirrographs of representative lesions at each of the four brain stem levels on the left side of the figure and corresponding lateral hypothalamic degenera- 
reticular origin of the pathway. As noted above, the hypothalamic degeneration was most dense and most consistantly apparent after lesions which destroyed the major cross-sectional area of the reticular formation rostral to the pons. While all the lesions damaged one or more structures peripheral to or within the reticular formation, damage to any given nonreticular nucleus or fiber system did not appear necessary for the induction of hypothalamic degeneration. For example, a number of mesencephalic lesions totally spared the central gray matter, raphe nuclei, and ventral tegmental region so that there was little or no apparent degeneration in the dorsal longitudinal fasciculus or medial forebrain bundle, but degenerated fibers entering the lateral hypothalamus through the subthalamus were still prominent. Furthermore, degeneration of the reticulohypothalamic pathway occurred in brains without damage to the cerebellorubrothalamic tract or the medial lemniscus.

\section{Discussion}

Our observations on the origin, trajectory, and termination of the reticulohypothalamic pathway correspond rather well to those of other workers using a variety of neuroanatomical techniques $(7,13,17)$. However, the other workers tended to report a more rostral extent of the system with fibers reaching forebrain structures such as olfactory tubercle, amygdala, and septum. We did not sec degencrated fibers in limbic forcbrain structures rostral to the optic chiasm in any of the brains of this series, but such negative results with degeneration techniques have little significance.

There have been reports of fibers originating in the pons (8) or spinal cord (1) and passing through the prerubral fields into the posterior hypothalamus. The relation between these fibers and those described in the present study is not clear. Further studies utilizing smaller lesions confined to specific portions of the reticular formation and applying electron microscopic methods to elucidate the nature of the synaptic endings in the hypothalamus are called for.

Another important question is whether there are reciprocal hypothalamoreticular fibers in the trajectory of the reticulohypothalamic pathway. Since it is the general case that diffuse brain stem pathways are bidirectional and specifically that both the dorsal longitudinal fasciculus and the medial forebrain bundle contain both ascending and descending fibers (10) one might expect to find descending fibers in this third system also. However, we have not seen such descending fibers in our studies to this time $(5,21)$.

tion stained by the Nauta method on the right side of the figure in the region of most dense degeneration indicated by the black rectangle in the inserted outline drawings. The shaded area in the outline drawing at " $\mathrm{A}$ " represents the rostral tip of the lesion. 
Two observations suggest that the reticulohypothalamic pathway, like other components of the ascending reticular system, is substantially polysynaptic and arises throughout the longitudinal extent of the upper (and possibly lower) brain stem reticular formation. First, there is a progressive diminution in the apparent density of the degeneration as the fibers course rostrally from a given lesion site. Second, the apparent density of the degeneration in a given lateral hypothalamic site diminishes with more caudal lesion placement. While this kind of data is hardly conclusive since the apparent density of degeneration may be affected by factors other than the number of degenerated fibers it is nevertheless strongly suggestive when considered in the light of the known morphology of the ascending reticular system $(11,17)$.

The apparent origin, morphology, and trajectory of the reticulohypothalamic pathway immediately suggest that it is related to the so-called extralemniscal sensory system and thus has the function of conveying polysensory information into the hypothalamus. Electrophysiological evidence shows conclusively that sensory information from a variety of modalities reaches the hypothalamus $(6,12,14,22)$. The latencies of the hypothalamic evoked responses are very long (20-50 msec) and thus implicate a polysynaptic conduction system. Feldman and co-workers (6), who have done the major work on hypothalamic sensory responses, found that the long latency evoked responses could be eliminated by lesions which destroyed the central tegmental region and concluded that these responses are mediated by a polysynaptic pathway through the reticular formation. ${ }^{3}$

Several workers have destroyed or stimulated specific ascending brain stem pathways in order to identify the pathways which carry afferent information to the hypothalamus for neuroendocrine reflexes. Lesions which included the central tegmental tract invariably blocked the reflexes; lesions involving the dorsal longitudinal fasciculus were usually but not invariably effective; and lesions involving the medial forebrain bundle or medial lemniscus were never effective in blocking the responses $(3,4,14,15)$. Studies in which the above brain stem pathways were electrically stimulated to induce neuroendocrine responses $(2,9,18,19)$ clearly support the conclusions gained from the lesion studies. Thus, several lines of evidence lead to the hypothesis that sensory information which modulates hypothalamic visceral and behavioral functions is conveyed by the reticulohypothalamic component of the ascending reticular system.

${ }^{3}$ R. Norgren and C. M. Leonard (taste pathways in rat brainstem, Science, in press) have recently found that third order gustatory fibers from the parabrachial area of the pons enter the central tegmental tract. It seems likely that gustatory pathway to the hypothalamus takes this route. 


\section{References}

1. Anderson, F. D., and C. M. Berry. 1959. Degeneration studies of long ascending fiber systems in the cat brain stem. J. Comp. Neurol. 111: 195-229.

2. Aulsebrook, L. H., and R. C. Holland. 1969. Central inhibition of oxytocin release. Amer. J. Physiol. $216: 830-842$.

3. Beyer, C. F., Mena, P. Pacheco, and M. Alcaraz. 1962. Blockage of lactation by brain stem lesions in the cat. Amer. J. Physiol. 202: 465-468.

4. Chang, H. C., J. J. Huang, Y. M. Lu, and Y. C. Tsang. 1940. A vagus-postpituitary reflex IX. General locus of the vagosupraoptic tract. Chin. J. Physiol. 15 : $445-464$.

5. EAger, R. P., C. C. ChI, and G. Wolf. 1971. Lateral hypothalamic projections to the hypothalamic ventromedial nucleus in the albino rat: Demonstration by means of a simplified ammoniacal silver degeneration method. Brain Res. 29 : 128-130.

6. Feldman, S. 1963. Effects of reticular formation lesions on afferent projections to the hypothalamus. Electroencephalogr. Neurophysiol. 15: 672-682.

7. Loizou, L. A. 1969. Rostral projections of noradrenaline containing neurones in the lower brain stem. J. Anat. 104: 593 (Abstr.).

8. McClure, T. D., and G. Clark. 1971. An ascending fiber projection pathway from the pontine reticular formation to the hypothalamus in the cat. Anat. Rec. $169: 371$.

9. Mills, E., and S. C. WANG. 1964. Liberation of antidiuretic hormone: Location of ascending pathways. Amer. J. Physiol. $207: 1399-1404$.

10. Nauta, W. J. H., and W. Haymaker. 1969. Hypothalamic nuclei and fiber connections, pp. 136-209. In "The Hypothalamus." W. Haymaker, E. Anderson, and W. J. H. Nauta, [Eds.]. Thomas, Springfield, Ill.

11. Nauta, W. J. H., and H. G. J. M. Kuypers. 1958. Some ascending pathways in the brain stem reticular formation, pp. 3-30. In "Reticular Formation of the Brain.” H. H. Jasper, L. D. Proctor, R. S. Knighton, W. C. Noshay, and R. T. Costello, [Eds.]. Little, Brown, New York.

12. Norgren, R. 1970. Gustatory responses in the hypothalamus. Brain Res. $21: 63-78$.

13. Reinoso-Suarez, F., and A. Llamas. 1968. Fibras ascendentes desde tegmento pontino oral en el raton. Acta Neurol. Latinoamer. 14 : 5-16.

14. Richard, P. 1970. An electrophysiological study in the ewe of the tracts which transmit impulses from the mammary glands to the pituitary stalk. J. Endocrinol. $47: 37-44$.

15. Richard, P., I. Urban, and R. Denamur. 1970. The role of the dorsal tracts of the spinal cord and of the mesencephalic and thalamic lemniscal system in the milk-ejection reflex during milking of the ewe. J. Endocrinol. 47: 45-52.

16. Scheiber, M. E., and A. B. Scheibel. 1966. Patterns of organization in specific and nonspecific thalamic fields, pp. 1316. In "The Thalamus." D. P. Purpura and M. Yahr [Eds.]. Columbia Univ. Press, New York.

17. Scheibel, M. E., and A. B. Scheibel. 1967. Anatomical basis of attention mechanisms in vertabrate brains, pp. 577-601. In "The Neurosciences." G. C. Quarton, T. Melnechuk, and F. O. Schmitt [Eds.]. Rockefeller Univ. Press, New York.

18. Tindal, J. S., G. S. Knaggs, and A. Turvey. 1967. The afferent path of the milk-ejection reflex in the brain of the guinea-pig. J. Endocrinol. 38 : 337-349. 
19. Tindal, J. S., G. S. Kraggs, and A. Turvey. 1969. The afferent path of the milk-ejection reflex in the brain of the rabbit. J. Endocrinol. 43: 663-671.

20. WoLf, G. 1968. Thalamic and tegmental mechanisms for sodium intake: Anatomical and functional relations to lateral hypothalamus. Physiol Behar. 3: 9971002.

21. Wolf, G., and J. Sutis. 1966. Fiber degeneration after lateral hypothalamic lesions in the rat. J. Comp. Neurol. $127: 137-156$.

22. Wykwicka, W., and M. H. Chase. 19j0. Projections from the buccal cavity to brain stem sites involved in feeding behavior. Exp. Nourol. 27 : 512-519. 